\title{
Mapping brain activity to predict stress responses
}

Hultman, R., et al. Cell 173, 1-15 (2018).

Consider a cohort of inbred laboratory mice. Genetically, they'll be nearly identical. But following a stressful event, some will carry on while others may begin to exhibit signs of a depression-like state. For all their variety, people aren't all that different: some seem resilient to stress, others susceptible. The disparity may be in the brain.

Researchers have been considering how activity in different brain regions contributes to vulnerability and resilience to stress in mice for a while now, but they had mostly looked at just one or two regions at a time, says Rainbo Hultman, a postdoctoral fellow studying the links between stress and depression in Kafui Dzirasa's lab at Duke University. Hints of an effect were there, but the picture was incomplete. A new study from the lab ups the ante: with the help of long-term, multi-circuit electrodes and some machine learning, Hultman, Dzirasa, and their collaborators were able to simultaneously follow electrical signaling between seven different regions of the mouse brain.
The team implanted electrodes into male C57BL/6J mice in areas previously hypothesized to contribute to depression in people and then recorded electrical activity before and after the animals were exposed to chronic social defeat stress, a paradigm for inducing depressive behavior. From all that data, the machine learning algorithm they developed with a team of engineers and statisticians was able to group which parts of the brain were engaged with one another, creating electrical maps, or 'electomes'.

Twenty five distinct maps emerged, with four correlating to vulnerability in stress-naïve mice (as determined by a choice interaction test following the chronic defeat). One in particular, Electome Factor 1, proved particularly predictive in mice exposed to three different stress paradigms: trauma via early maternal separation; physiological manipulation with interferon-alpha; and genetic manipulation of a known susceptibility gene. When testing potential interventions though, like deep-brain stimulation and the drug ketamine, the researchers saw no change in Electome Factor 1 activity; Factors 2 and 3, however, did respond. Thus, vulnerability to depression and the treatment of it after it develops seem to be influenced by different underlying mechanisms in the brain-an interesting result in light of growing literature that's teasing apart neural connectivity in human patients with depression and re-defining subsets of the disease accordingly, says Hultman.

Hultman, a molecular biologist, hopes to look in to the molecular underpinnings of these circuits in the future, while Dzirasa is interested in the idea of a pacemaker for the brain that can 'fix' broken connections. There's still more brain to consider too, but nevertheless, the electrical maps represent a new way of considering stress.

\section{Ellen P. Neff}

Published online: 23 May 2018

https://doi.org/10.1038/s41684-018-0077-9

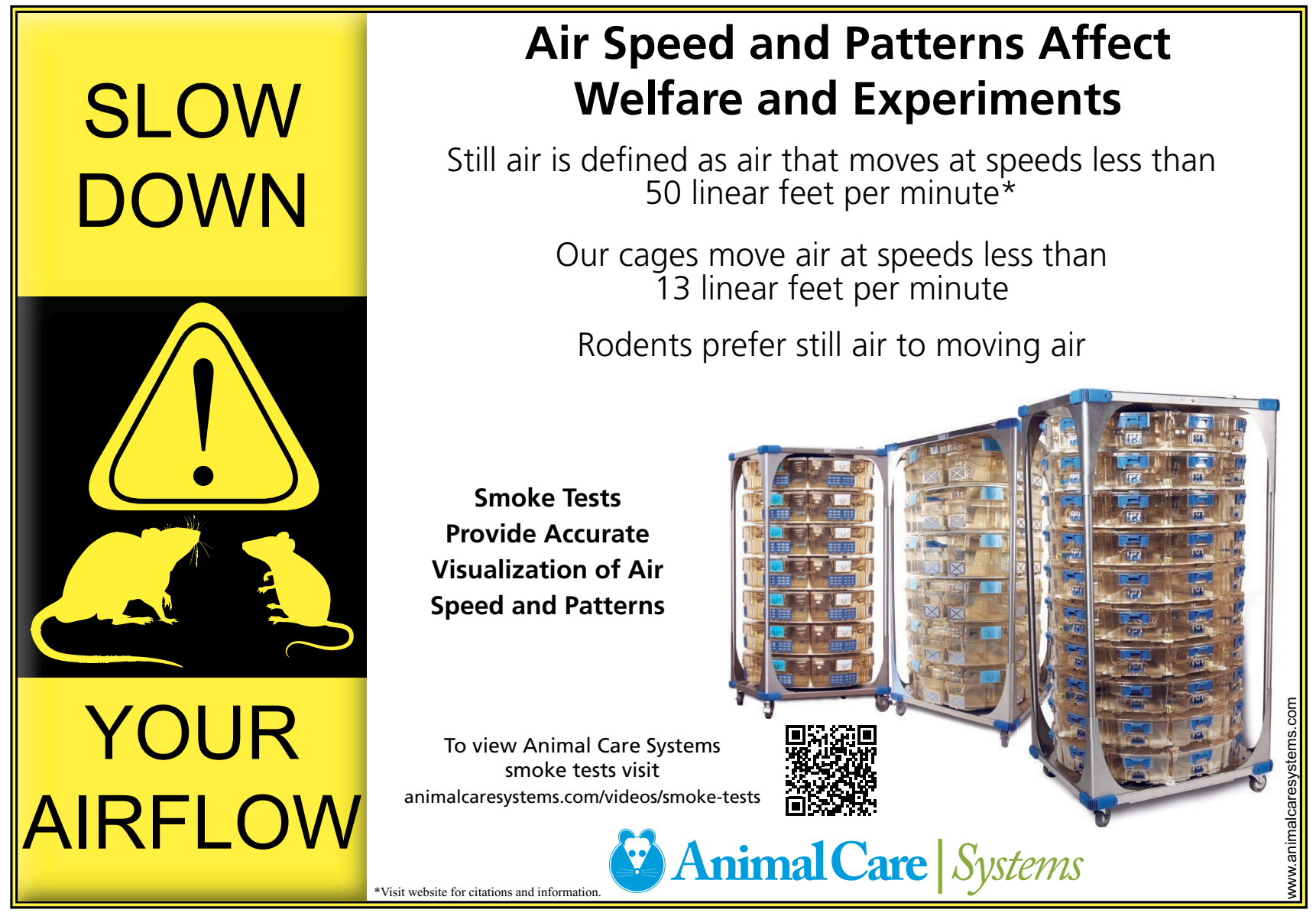

